\title{
Composition and Antimicrobial Activity of Mangifera Indica L.Sap
}

\author{
Subburaj.M \\ Regional Analytical Laboratory, Florican Hills, Calicut,Kerala, India 673009. \\ subuchem@gmail.com
}

ABSTRACT : The unripe Mangifera indica (Mango) sap was collected from Western ghat in South India. The sap was separable into two layers as aqueous and organic. Analysis of the organic layer by gas chromatography $(G C)$ and Gas chromatography with Mass spectrometry (GC-MS) revealed the identification of 28 compounds out of 30 compounds accounting 98.49\% of the total organic layer. The sap was found to be rich in monoterpenes with major constituents being myrcene (72.78\%), a-Farnesene (5.88\%), a-copaene (4.14\%) and Farnesyl acetone (3.28\%). The identified compounds are comparing with earlier studies. High amount of $\alpha$-Farnesene is present in the western ghat mango variety compare with other mangoes. Our study supports the earlier finding of the sap is responsible for flavour, taste and the sap essential oil composition is depends on varieties. Antimicrobial study using disk diffusion method and Minimum inhibitory concentration (MIC) was carried out. The organic layer showed strong in vitro growth inhibition activity against Grampositive bacteria and moderate to least level activities in gram negative and fungi at different levels of concentrations among the tested microorganisms, According to MIC they exhibited strong antibacterial activity against Pseudomonas aeruginosa $(M I C=100 \mu \mathrm{g} / \mathrm{ml})$ and moderate activity against Staphylococcus albus, Klebsiella aerogenes, Proteus vulgaris $(M I C=800 \mu \mathrm{g} / \mathrm{ml})$ and no activity against streptococcus faecalis. The antimicrobial activity of Mangifera indica sap against the different tested microorganisms may be attributed. Hence, the Mangifera indica sap may be used as a preservative in food industry, because of it is more economical when compared to other preservatives. Also they would cause no health hazards to consumers. With these advantages Mangifera indica sap could be suggested for food preservative particularly in pickle industry.

Key words: Mango Sap, chemical composition, Antimicrobial activity, Preservatives

\section{I.INTRODUCTION}

Mangoes belong to the genus Mangifera of the family Anacardiaceae. Most of the fruit trees that are commonly known mangoes belong to the species Mangifera indica. The other edible Mangifera species generally have lower quality and are commonly referred as wild mangoes. There are over one thousand mango varieties throughout the world, which is a testament to their value to human kind. Mangoes have been grown throughout the tropical and subtropical world, for thousands of years and have become an integral part of many cultures (1). Many species of Mangifera indica have been widely used as medicines, food and flavor industries etc. The young and unripe mangoes are acidic and taste it is utilized for various culinary purposes. The tender mangoes were used in wide spectrum of household remedies. The mango sap has been used to treat the pain of bee and scorpion strings. Many of the traditional Indian medicinal uses of mango involve eating unripe fruits. The drink made from unripe mango fruit is used as a remedy for exhaustion and heat stroke. Half ripe fruit eaten with salt and honey is used for the treatment of gastro-intestinal disorder, bilious disorders, blood disorders and scurvy. The mango stalk is generally used to cleaning the teeth and fresh leaves masticated to tone up the gums. So the mango leaf powders are used in most of the ayurvedic tooth powders. It should be noted that unripe fruit contains toxic sap that when eaten in excess can cause throat irritation, indigestion, dysentery and colic. Diabetes has been treated with a drink made from the infusion of fresh mango leaves. Dried mango seeds ground into flour are used to treat diarrhea. Diarrhea and throat disorders are treated by gargling bark extracts mixed with water. The kernel juice is snuffed once a day for three days to stop nasal bleeding. The rind is astringent and is a stimulating tonic in debility of stomach.. In Samoa, a bark infusion has been a traditional remedy for mouth infections (Pala gutu).(2) In Tonga infusion of mango leaves, orange (Citrus sinensis) and other species mixtures are used to treat relapse sickness (Kita).(3) The fruits and its by-products are used for animal fodder. Today mango and its flavor were added to many products and its by-products. 


\section{EXPERIMENTAL WORK}

Mangifera indica unripe sap (mango sap) that spurts and oozes from the abscission zone of the peduncle was collected in a stopper test tube after destalking during the winter season from the western ghat in south India at the stage of three moths from flowering. The sap may cause dermatitis, rash and blistering while contact with skin so necessary safety precautions should be taken while collecting the sap. The collected sap in a test tube slowly separates into clearly separable two layers as aqueous and organic. The sample was kept under 40C until the analysis and bioassays test.

GC ANALYSIS

Gas chromatography (GC) analysis were carried out using a GC-14A with FID and C-R6A-Chromatopac integrator (Shimadzu, Japan), a GC-3700 with FID (Varian,Germany) and C-R1B-chromatopac integrator (Shimadzu). The carrier gas was hydrogen, the injector temperature $2500 \mathrm{C}$ and the detector temperature 3200C. The temperature program was; $400 \mathrm{C} / 5 \mathrm{~min}$ to $2800 \mathrm{C} / 5 \mathrm{~min}$, while a heating rate of $60 \mathrm{C} / \mathrm{min}$. The columns were a $30 \mathrm{mX} 32 \mathrm{~mm}$ bonded apolar OV-1-column and 30mX0.32mm bonded FSOT-RSL-200 fused silica, with a film thickness of $0.25 \mu \mathrm{m}$ (Biorad,Germany) and $30 \mathrm{X} 0.32 \mathrm{~mm}$ bonded stabilwax, with a film thickness of $0.50 \mu \mathrm{m}$ (Restek, USA). Quantification was achieved using peak area calculations (on apolar column) and by co-injection of pure compounds and compounds identification was partially carried out using correlations between retention times ( 4-6)

GC/MS ANALYSIS

Gas chromatography with mass spectrometry (GC/MS) analysis were carried out by GC -17A with QP 5000 (Shimadzu) and Compaq-proLinea data system (Class5k-software),a GC-HP5890 with HP5970-MSD (Hewlett-packard,USA0 and Chemstation software on a Pentium PC (Bohm,Austria), a GCQ (Finniganspectronex, Germany-USA) and Gateway-2000-PS75 data system(Siemens-Nixdorf,Germany,GCQ-software) were used. The carrier gas was helium, the injector temperature $2500 \mathrm{C}$, interface heating was at $3000 \mathrm{C}$ and ionsource-heating at 2000C;EI-model was 70ev, and the scan range was 41-450amu. For other parameters, see the description of GC/FID, above. Mass spectra correlation were done using Wiley, NBS, NIST and our own library on-line as well as using published mass spectral data off-line (4,7-9).

MICROBIAL STRAINS AND MEDIA

The antibacterial activity of the essential oil against four Gram positive bacteria Staphylococcus aureus, Bacillus subtilis, streptococcus faecalis, Staphylococcus albus and four gram negative bacteria Escherichia coli, Pseudomonas aeruginosa, Klebsiella aerogenes, Proteus vulgaris and the antifungal activity against the pathogenic fungi Candida albicans and Aspergillus niger. All the microorganism were obtained from National Chemical Laboratory (NCL), Pune , India and maintained by periodic sub-culturing on Nutrient agar and sabouraud dextrose medium. The culture medium used for bacteria was Nutrient agar, whereas sabouraud dextrose agar was used for fungi. Media were sterilized by autoclaving at $1200 \mathrm{C}$ for $15 \mathrm{~min}$ and all subsequent manipulations were carried out in a laminar flow cabinet.

ANTIMICROBIAL ASSAYS

Antimicrobial activities of the mango sap were determined by employing the standard disk diffusion technique (13) before experimentation, the sap was diluted with DMSO in water (4\%) at different concentration ranges from $5 \mathrm{mg} / \mathrm{ml}$ to $100 \mu \mathrm{g} / \mathrm{ml}$. Whatman-1 filter paper disks from $6 \mathrm{~mm}$ diameter, placed in dry Petri dishes, were autoclaved. These sterile Whatman-1 filter paper disks were added to each of the test sap and shaken thoroughly. The filter paper disks were allowed to dry and were carefully placed over the spread cultures and incubated at $370 \mathrm{C}$ for 24 hours for bacteria and at $28-300 \mathrm{C}$ for 48 hours for fungi. Paper disks treated with antimicrobial standards alone served as control. The definite zone of inhibition of any dimension surrounding the paper disks were measured accurately to the nearest millimeter by means of a metric ruler and an illuminated colony counter. The minimum inhibitory concentrations (MIC) were determined as the lowest concentrations. Standard antibiotic ciprofloxacin $2 \mu \mathrm{g} / \mathrm{disc}$ used as a control the sensitivity of the tested bacteria, whereas clotrimazole $10 \mu \mathrm{g} / \mathrm{disk}$ is used as control against the tested fungi.

\section{III.RESULTS AND DISCUSSION}

The qualitative and quantitative studies of Mangifera indica sap by GC and GC/MS are shown in Table-1. Thirty compounds were determined and twenty eight compounds were identified representing about $98.48 \%$ of the total essential oil. The essential oil in the sap mainly composed of myrcene (72.78\%), $\alpha$-Farnesene $(5.88), \alpha-$ Copaene $(4.14 \%)$ and Farnesyl acetone $(3.28 \%)$. It is interesting to note that the compound myrcene is present in almost all mango varieties (14-17). Higher percentage of $\alpha$-Farnesene is reported first time in mango sap which is present only in Kerala cultivar (15).

The combination of E,E Farnesol along with Z,E Farnesol, Myrcenol altogether and the combination of 
Trans $\beta$-Ocimene, Cis- $\beta$-Ocimene and Caryophyllene along with Caryophyllene oxide presence is reported in our studies which does not reported in any past studies in Mangifera indica sap. The green aroma note typical of mango was correlated to monoterpene hydrocarbons (16). Earlier studies and Olfactory evaluation showed the presence of Trans $\beta$-Ocimene, Cis- $\beta$ - Ocimene have a floral herbaceous odor and Myrcene,(Z)- $\beta$-Ocimene were reported to be responsible for the green aroma of raw mango and its present in most of the mango varieties including our samples supports their findings. (17). Terpinolene as a major component in the Kensington variety $(83.7 \%)$ and car-3-ene as a major component in Irwin variety (89.8\%). Terpinolene, Car-3-ene were dominant compounds in several mango varieties but these compounds does not found in our sample and most of the mangoes in Indian cultivar. It is known that the composition of mango sap vary depending upon the variety (1419). The major constituent of the essential oil influence the taste of the mango which is evident for the sap exudates from Totapuri, Raspuri, Seedlings and malgoa is Myrcene whereas in Alphonso (Badami) and Banganapalli is Ocimene and Mallika the major constituent is Limonene. The taste of the Totapuri, Raspuri, Seedlings and Malgoa is almost same with the Western Ghats local variety.

\section{ANTIMICROBIAL ACTIVITY}

Mangifera indica sap showed strong antimicrobial activity against the Gram positive bacteria such as Staphylococcus aureus, Staphylococcus albus and Gram negative bacterial Proteus vulgaris and good in pathogenic fungi Candida albicans at concentration range of $1 \mathrm{mg} / \mathrm{ml}$ with inhibition activities of $12 \mathrm{~mm}$. Streptococcus faecalis remain unaffected by the mango sap. The oil also shown activity against B. Subtilis, Pseudomonas aeruginosa, Klebsiella aerogenes $(11 \mathrm{~mm})$ Gram negative bacterial E. coli and Fungi A. niger show strong antimicrobial activity in higher concentrations $2.5 \mathrm{mg} / \mathrm{ml}$. These results could be comparable with the similar range produced by the standards which is tabulated in Table 2, meanwhile the MIC's of the mango sap against several bacteria and fungi are presented in Table 3. As shown in this table the mango sap has variable levels of inhibition. Pseudomonas aeruginosa $(100 \mu \mathrm{g} / \mathrm{ml})$ and Staphylococcus albus, Klebsiella aerogenes, Proteus vulgaris (MIC $=800 \mu \mathrm{g} / \mathrm{ml}$ ) are the most sensitive micro organism to the mango sap because of their low MIC values ranging from high concentration to low. The essential oil has the same MIC`s $(1 \mathrm{mg} / \mathrm{ml})$ against the Staphylococcus aureus, Candida subtilis, Candida albicans. Also the highest MIC values are determined against E.coli and Aspergillus niger $(2.5 \mathrm{mg} / \mathrm{ml})$. No activities are seen in S. Faecalis.

\section{CONCLUSION}

The antimicrobial activity of Mangifera indica sap against the different tested microorganisms may be attributed. Hence, the Mangifera indica sap may be used as a preservative in food industry. These sap are more economical when compared to other preservatives. Also they would cause no health hazards to consumers. With these advantages Mangifera indica sap could be suggested for food preservative. In Kerala Tender mangoes are picking with short stems $(<5 \mathrm{~cm})$ with a stick, viscous sap for making pickles so that the sap is retained inside. This practice helps in preventing bacterial and fungal infections of mango pickles, so that it can be kept for a long period without adding any preservatives. This practice might be helpful in pickle industry because of the sap can produce good aroma and can act as a good preservative. Mango varieties like Totapuri, Vadumaangai, Aavakkai, seedlings are very famous for mango preparation or pickled compare to the popularly used ripe fruits like Banganapalli, malgoa and Raspuri which have low yield of essential oil compare to the former. So the mango contain more essential oil is useful for mango preparation instead of raw eating.

1. Bompard, J.M ., Acta Horticulture., 341, 1993,P 69-77.

\section{REFERENCES}

2. Whistler,W.A., Samoan herbal medicine Isle Botanica, Honolulu, 1996,116-120.

3. Whistler,W.A., Tongan herbal medicine Isle Botanica, Honolulu, 1992,122-125.

4. Giddings., J.C., J.Chromatogr., 3, 1995,A703.

5. 5.kondjoyan,N., berdaque,J.,L. A compilation of relative retention Indices for the Analysis of Aromatic compounds, Edition du laboratoire Flaveur,Saint Genes Champanelle., 1996.

6. Jennings,W., Hibamoto, T., Qualitative analysis of flavour and Fragrance by Glass capillary with GC., Academic Press., 1980.

7. Cornu,A.,m massot,R., Compilation of mass spectral data., London: heyden \& Sons, 1975, Vol.1-2.

8. Eight Peak index of Mass Spectra,Mass Spectrometry Data centre (Distribution),Reading -1970,Vol.1-2.

9. Mclafferty,F.W., Staufer,D.B., The Wiley NBS Registry of Mass Spectral Data,Newyork: John Wiley., 1974,Vol .II.

10. Schmaus,G., Thesis., University of Würtzburg., Germany., 1988.

11. Stenhagen,E., Abrahamsso,S., and Mac Lafferty, F.W., Registry Mass spectral Data., new York:John Wiley., 1974,Vol.1-4.

12. Wealth of India; Raw materials., CSIR.,New Delhi., Vol XI., 1976, 211-222. 